\title{
Liver Imaging Reporting and Data System:an expert consensus statement
}

This article was published in the following Dove Press journal:

Journal of Hepatocellular Carcinoma

17 February 2017

Number of times this article has been viewed

\section{Khaled M Elsayes' \\ Ania Z Kielar ${ }^{2}$ \\ Michelle M Agrons ${ }^{3}$ \\ Janio Szklaruk' \\ An Tang ${ }^{4}$ \\ Mustafa R Bashir ${ }^{5}$ \\ Donald G Mitchell ${ }^{6}$ \\ Richard K Do ${ }^{7}$ \\ Kathryn J Fowler ${ }^{8}$ \\ Victoria Chernyak ${ }^{9}$ \\ Claude B Sirlin ${ }^{10}$}

'Department of Diagnostic Radiology, The University of Texas MD Anderson Cancer Center, Houston, TX, USA; ${ }^{2}$ Department of Diagnostic Radiology, University of Ottawa, Ottawa, ON, Canada; ${ }^{3}$ Department of Diagnostic Radiology, Baylor College of Medicine, Houston, TX, USA; ${ }^{4}$ Department of Radiology, Radio-Oncology and Nuclear Medicine, Université de Montréal, Montreal, QC, Canada; ${ }^{5}$ Department of Diagnostic Radiology, Duke University School of Medicine, Durham, NC,

${ }^{6}$ Department of Diagnostic Radiology, Thomas Jefferson University, Philadelphia, PA, ${ }^{7}$ Department of Radiology, Memorial Sloan Kettering Cancer Center, New York, NY, ${ }^{8}$ Mallinckrodt Institute of Radiology, Washington University in Saint Louis, Saint Louis, MO, ' Department of Radiology Albert Einstein College of Medicine, Bronx, New York, NY, ${ }^{10}$ Department of Diagnostic Radiology, University of California, San Diego, CA, USA

Correspondence: Khaled M Elsayes Department of Diagnostic Radiology, The University of Texas MD Anderson Cancer Center, 1400 Pressler Street, Houston, TX 77030 , USA

Tel + I $7 / 37453025$

Fax +I 7I3 7944379

Email kmelsayes@mdanderson.org
Abstract: The increasing incidence and high morbidity and mortality of hepatocellular carcinoma (HCC) have inspired the creation of the Liver Imaging Reporting and Data System (LIRADS). LI-RADS aims to reduce variability in exam interpretation, improve communication, facilitate clinical therapeutic decisions, reduce omission of pertinent information, and facilitate the monitoring of outcomes. LI-RADS is a dynamic process, which is updated frequently. In this article, we describe the LI-RADS 2014 version (v2014), which marks the second update since the initial version in 2011.

Keywords: hepatocellular carcinoma, imaging, reporting, cirrhosis, hyperenhancement washout

\section{Introduction}

Hepatocellular carcinoma (HCC) is the fifth most common tumor and the second most common cause of cancer-related death worldwide. ${ }^{1}$ The incidence of HCC in the US has tripled over the last three decades, ${ }^{2}$ which has been attributed largely to the epidemic of chronic hepatitis $\mathrm{C}$ virus (HCV) infection acquired through intravenous drug use and blood transfusions between 1960 and $1980 .^{2-4} \mathrm{HCV}$ infection accounts for the increasing incidence of HCC in developed countries and has become the single most frequent cause of $\mathrm{HCC},{ }^{5}$ although $\mathrm{HCV}$ is associated with only one-third of $\mathrm{HCC}$ cases in developing countries. Thus, hepatitis B virus (HBV) infection still accounts for more than half of the world's overall HCC burden, although its incidence is now decreasing because of increasing worldwide vaccination. ${ }^{2}$

The most consistent predisposing factor in the development of $\mathrm{HCC}$ is cirrhosis, as $80 \%$ of HCC cases develop in the cirrhotic liver. ${ }^{6}$ In addition to HCV and HBV infections, other causes of cirrhosis include hereditary hemochromatosis, alcoholic cirrhosis, biliary cirrhosis, and now increasingly, nonalcoholic steatohepatitis (NASH) related to the rising incidence of obesity, diabetes, and metabolic syndrome. ${ }^{7}$ It has also been suggested that most cases of cryptogenic cirrhosis represent the end stages of nonalcoholic fatty liver disease in which the liver has progressed to a markedly fibrotic state devoid of fat. ${ }^{8}$ In contrast to other causes of cirrhosis, $30 \%$ of patients with chronic HBV infection develop HCC without cirrhosis. ${ }^{9}, 10$

The significant morbidity and mortality associated with HCC makes early detection and diagnosis critical. Serum alpha-fetoprotein (AFP) measurements and ultrasound have been associated with greater mortality reduction than other screening methods, although their sensitivity is only 50-60\% individually. ${ }^{11,12}$ Contrast-enhanced magnetic resonance imaging (MRI) and computed tomography (CT) are the two most widely used 
imaging techniques for diagnosis of HCC following initial detection by surveillance AFP or ultrasound, with per-lesion sensitivities of $83 \%$ and $76 \%$ and per-lesion specificities of $87 \%$ and $89 \%$, respectively. ${ }^{13}$ As important advancements in imaging technology have evolved, imaging has played an increasingly important role in HCC evaluation, so that pretreatment biopsy is not currently mandated by most current clinical practice guidelines in a patient at risk for HCC when appropriate imaging demonstrates the typical features of HCC. ${ }^{14}$ At the other extreme, incorrect diagnosis can adversely affect management if a false-negative diagnosis leads to delayed detection until advanced stages or if falsepositive diagnoses may lead to unnecessary surgery or treatment. The detection of small tumors and the management of small hypervascular nodules remain important challenges in imaging diagnosis and evaluation of $\mathrm{HCC}{ }^{15}$

With imaging occupying a central role in diagnosis, staging, and management decisions, the need for a consistent lexicon and well-defined diagnostic criteria has never been greater. To address this need and to improve clarity and quality in diagnostic reports, the Liver Imaging Reporting and Data System (LI-RADS), a consensus American College of Radiology (ACR)-supported initiative analogous to BI-RADS in breast imaging, was created. ${ }^{16}$ LI-RADS aims to reduce variability in exam interpretation, improve communication, facilitate clinical therapeutic decisions, reduce omission of pertinent information, and facilitate the monitoring of outcomes. ${ }^{16}$

LI-RADS was created by radiologists collaborating with other liver specialists, for all radiologists to use, in both academic and community or private practice settings. LIRADS is updated continuously, incorporating improvements in imaging techniques. The LI-RADS 2014 version (v2014), described in this article, marks the second update since the initial version was released by the ACR in 2011, offering several enhancements to the previous version (Table 1$).{ }^{17}$ These refinements are discussed in later sections of this article. As part of LI-RADS, there is an Evidence Based Workgroup that regularly reviews new publications and information in the literature and helps to guide future versions of LI-RADS. A 2017 update is planned with further refinements, improvements, and updates; some of these future expanded roles of LI-RADS v2017 will be discussed later.

\section{LI-RADS: overview}

LI-RADS is a system created for the standardized interpretation and reporting of liver imaging examinations in patients at risk for HCC. This system was developed with the cooperative and ongoing efforts of an ACR-supported committee composed of diagnostic radiologists with expertise in liver imaging, with valuable input from hepatobiliary surgeons, hepatologists, hepatopathologists, and interventional radiologists. The goal of LI-RADS is to provide standard terminology and diagnostic criteria to help readers assign categories (from 1 through 5), which reflect the probability of benignity or malignancy in patients at risk for HCC. ${ }^{17}$ LI-RADS also provides recommendations regarding technical requirements of contrast-enhanced CT and MRI. As part of the LI-RADS mission, this system provides on the ACR website a lexicon of imaging features illustrated by schematics and an atlas lexicon of terminology to allow standardized language to be used in radiology reports. v2014 included a standardized downloadable reporting template to help radiologists include all pertinent findings and follow the LI-RADS terminology and approach.

Classification within the LI-RADS system is dependent on distinct imaging features, which increase or decrease the probability of HCC to various degrees, using an algorithmic approach. ${ }^{16}$ This begins by noting whether any distinctive focal alteration within liver parenchyma is present, referred to as an observation, because not all of these are masses. The process by which liver observations are classified is demonstrated in the algorithm in Figure $1 .{ }^{17}$ Unlike the previous version, v2014 includes a distinct category for previously treated observations. ${ }^{17}$ Of note, imaging criteria in v2014 applies to CT or MRI with the use of extracellular contrast agents and also includes material on the interpretation of MRI performed with hepatobiliary contrast agents.

Similar to LI-RADS for CT and MRI, CEUS LI-RADS was more recently developed as a standardizing system for technique, interpretation, reporting, and data collection of contrast-enhanced ultrasound in patients at risk for developing HCC. ${ }^{17}$ Another impressive collaboration of national and international radiology and hepatology experts, the CEUS LI-RADS algorithm was first released in June 2016, shortly after US Food and Drug Administration approval of the use of ultrasound contrast. ${ }^{17}$ With the development of CEUS LI-RADS guidelines, contrast-enhanced ultrasound provides another modality with which HCC can be diagnosed, characterized, and treated sooner and more efficiently, without the need for pretreatment biopsy. Although contrast-enhanced ultrasound is not within the scope of this article, we feel it is important to note that, like LI-RADS for CT and MRI, CEUS LI-RADS is a dynamic process which is scheduled for updates in 2017, and 2020, and serves as a valuable resource in the improvement of patient care. 
Table I Comparison between LI-RADS v20 II and v20I4

\begin{tabular}{lll}
\hline Category & LI-RADS v20I I & LI-RADS v20I4 \\
\hline LR treated & No LR-treated category & Any observation that has undergone locoregional treatment, \\
& No difference between LI-RADS V20I I and LI-RADS v20I4 & No difference between LI-RADS V20I I and LI-RADS v20I4 \\
LR-I and LR-2 & $<20$ mm arterial-phase iso- or hypoenhancing mass & $<20$ mm arterial-phase iso- or hypoenhancing with "washout" \\
LR-3 & with $\leq$ I of the following: portal/delayed phase & only or "capsule" only or threshold growth only or none \\
Intermediate & hypoenhancement, diameter increase by $\geq 10$ mm in I year & $\geq 20$ mm arterial-phase iso- or hypoenhancing without \\
probability for HCC & $<20$ mm arterial hyperenhancing mass with neither portal/ & "washout," "capsule," or threshold growth \\
& delayed phase hypoenhancement nor $\geq 10$ mm diameter & $<20$ mm arterial-phase hyperenhancing without "washout," \\
& increase within I year, or $<20 \mathrm{~mm}$ arterial hyperenhancing & "capsule," or threshold growth
\end{tabular}

LR-4

Probably HCC

LR-5

Definitely HCC

LR-5V

LR-M nonmass-like and stable

$\geq 20 \mathrm{~mm}$ iso- or hypoenhancing mass with neither portal/

delayed phase hypoenhancement nor $\geq 10 \mathrm{~mm}$ diameter

increase within I year, or $\geq 20 \mathrm{~mm}$ arterial hyperenhancing

nonmass-like and stable

LR-4A

$<20 \mathrm{~mm}$ arterial iso- or hypoenhancing mass with both portal/delayed phase hypoenhancement and $\geq 10 \mathrm{~mm}$ diameter increase within I year

$<20 \mathrm{~mm}$ arterial-phase hyperenhancing mass with either portal/delayed phase hypoenhancement or $\geq 10 \mathrm{~mm}$ diameter increase within I year, but not both LR-4B

$\geq 20 \mathrm{~mm}$ arterial-phase iso- or hypoenhancing mass with portal/delayed phase hypoenhancement or $\geq 10 \mathrm{~mm}$ diameter increase within I year or both

$\geq 20 \mathrm{~mm}$ arterial-phase hyperenhancing mass with neither portal/delayed phase hypoenhancement nor $\geq 10 \mathrm{~mm}$ diameter increase within I year

LR-5A

$\geq 10$ and $<20 \mathrm{~mm}$ arterial hyperenhancing mass with both portal/delayed phase hypoenhancement and $\geq 10 \mathrm{~mm}$ diameter increase within I year

LR-5B

$\geq 20 \mathrm{~mm}$ arterial hyperenhancing mass with portal/delayed phase hypoenhancement or $\geq 10 \mathrm{~mm}$ diameter within I year or both

No LR-5V category

No LR-M category
No A or B distinction

$<20 \mathrm{~mm}$ arterial-phase iso- or hypoenhancing with $\geq 2$ of the following: "washout," "capsule," or threshold growth $<10 \mathrm{~mm}$ arterial-phase hyperenhancing with $\geq 1$ of the following: "washout," "capsule," or threshold growth 10-19 mm arterial-phase hyperenhancing with "washout" only or "capsule" only or threshold growth only

$\geq 20 \mathrm{~mm}$ arterial-phase iso- or hypoenhancing with $\geq 1$ of the following: "washout," "capsule," or threshold growth $\geq 20 \mathrm{~mm}$ arterial hyperenhancing mass without "washout", "capsule", or threshold growth

No $A$ or $B$ distinction

10-19 mm arterial-phase hyperenhancing with $\geq 2$ of the following: "washout," "capsule," or threshold growth $\geq 20 \mathrm{~mm}$ arterial hyperenhancing with $\geq 1$ of: "washout," “capsule," or threshold growth

Mass with definite tumor in vein

Features suggestive of non-HCC malignancy such as rim arterialphase hyperenhancement or peripheral washout appearance

Notes: See Table 2 for LI-RADS classifications.

Abbreviations: LI-RADS, Liver Imaging Reporting and Data System; HCC, hepatocellular carcinoma.

\section{LI-RADS algorithm}

The LI-RADS algorithm is utilized from top to bottom and left to right. Treated observations - according to clinical history - are assigned to the LI-RADS-treated category. Untreated observations may be assigned to the following categories: LR-1 for definitely benign, LR-2 for probably benign, LR-M for probable malignancy not specific for HCC, and LR-5V for HCC with tumor in vein. Observations that do not fit in any of the previous categories will be assigned to one of the following categories: LR-3 for intermediate probability of HCC, LR-4 for probable HCC, or LR-5 for definite HCC depending on the combination of major imaging features discussed subsequently (Table 2 ). ${ }^{17}$

\section{Major imaging features}

In v2014, LI-RADS uses five major imaging features to establish the diagnosis of HCC. These include arterial-phase hyperenhancement, diameter, washout appearance, capsule appearance, and threshold growth. ${ }^{17}$

v2014 no longer requires formal identification of a mass, which was previously defined as a three-dimensional spaceoccupying lesion that displaces or replaces underlying hepatic parenchyma. ${ }^{16}$

\section{Arterial-phase hyperenhancement}

Arterial-phase hyperenhancement, a major feature of HCC diagnosis, reflects the increased arterial vascularization that 


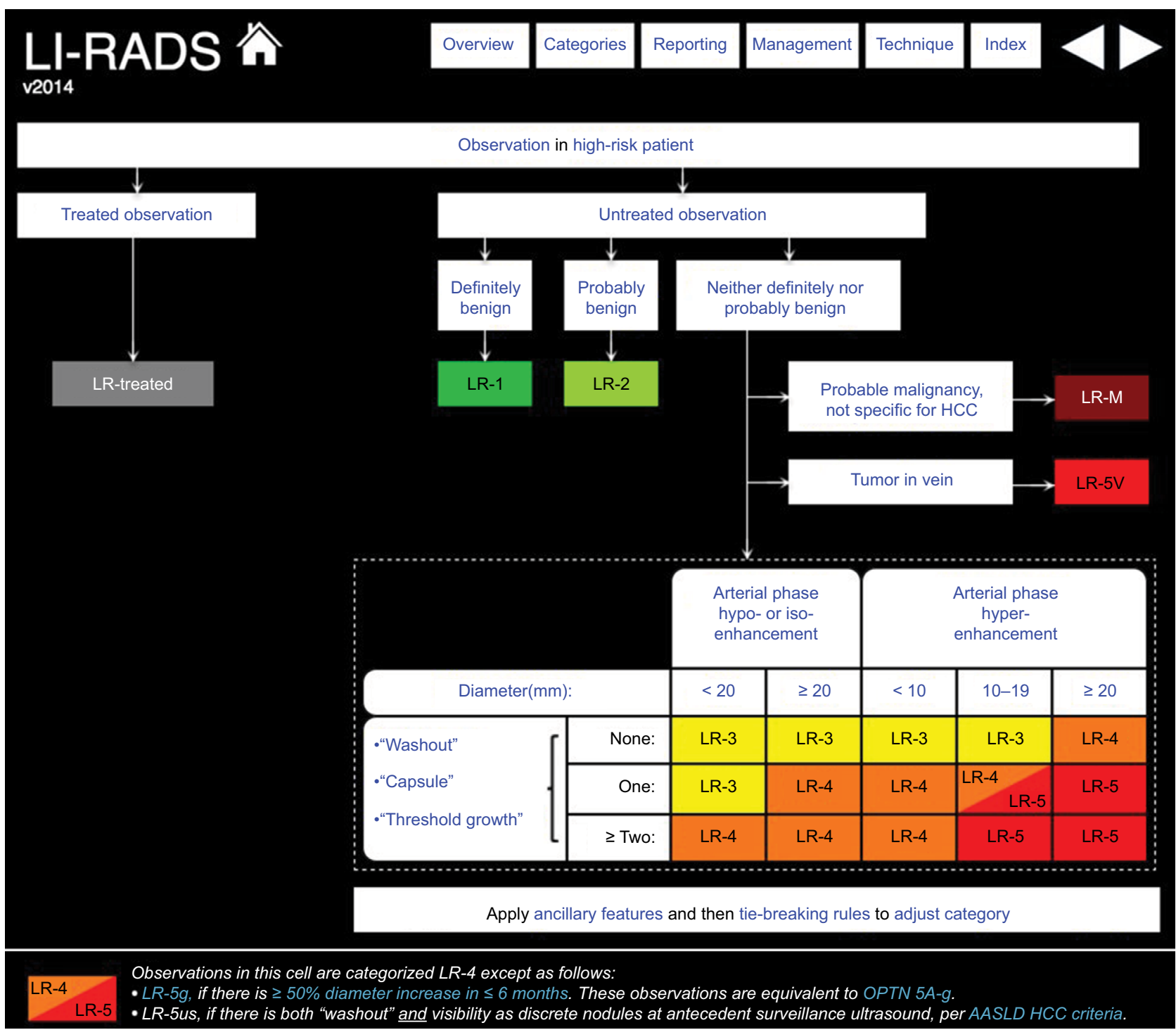

Figure I LI-RADS v20I4 algorithm.

Notes: See Table 2 for LI-RADS classifications. Copyright @2016. Dove Medical Press. Reproduced from American College of Radiology. Liver Imaging and Reporting System version 2014. Available from: https://nrdr.acr.org/lirads/. ${ }^{17}$

Abbreviations: LI-RADS, Liver Imaging Reporting and Data System; HCC, hepatocellular carcinoma; OPTN, Organ Procurement and Transplantation Network; AASLD, American Association for the Study of Liver Diseases.

Table 2 LI-RADS classification

\begin{tabular}{ll}
\hline Category & Description \\
\hline LR-I & Definitely benign \\
LR-2 & Probably benign \\
LR-3 & Intermediate probability of HCC \\
LR-4 & High probability of HCC, not I00\% \\
LR-5 & Definitely HCC \\
LR-5V & Definite venous invasion regardless of other \\
& imaging features \\
LR treated & LR-5 lesion status post-locoregional treatment \\
LR-M & Non-HCC malignancies that may occur in cirrhosis: \\
& metastases, lymphoma, cholangiocarcinoma, PTLD \\
\hline
\end{tabular}

Abbreviations: LI-RADS, Liver Imaging Reporting and Data System; HCC, hepatocellular carcinoma; PTLD, post-transplant lymphoproliferative disorder.

occurs at the expense of portal venous supply during hepatocarcinogenesis. ${ }^{18-20}$ Only observations with arterial-phase hyperenhancement can be categorized as LR-5 (definite
HCC). ${ }^{17}$ All or part of the observation in question must demonstrate greater enhancement, and have higher signal intensity, than the surrounding liver during the arterial phase (Figure 2). ${ }^{20,21}$ The late arterial phase is preferred over the early arterial phase to demonstrate this major feature.

Arterial-phase hypoenhancement or isoenhancement refers to enhancement of an observation that is less than or equal to that of the liver during the arterial phase. ${ }^{16}$ This feature does not include nonenhancing observations. ${ }^{17}$ If definitive discrimination between hyperenhancement and iso- or hypoenhancement cannot be made, then this cannot be considered hyperenhancement. After evaluating the presence or absence of hyperenhancement, other major features - observation diameter, washout appearance, capsule appearance and threshold growth - are then used to assign an LI-RADS category. ${ }^{17}$ 

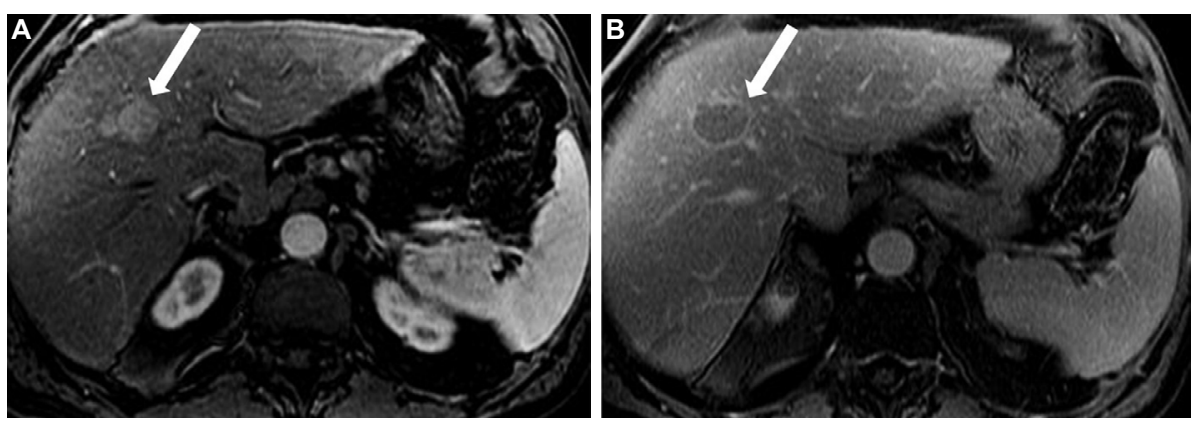

Figure $2 \mathrm{HCC}$ in a 57-year-old man with chronic HCV.

Notes: Axial post-contrast TI-weighted MR images in arterial (A) and delayed phase (B) demonstrate a well-circumscribed oval lesion measuring $2.5 \mathrm{~cm}$ in maximal dimension exhibiting homogeneous hyperenhancement in the arterial phase (arrow) with washout and enhancing capsule in the delayed phase (arrow). This lesion is category LR-5. See Table 2 for LI-RADS classifications.

Abbreviations: $\mathrm{HCC}$, hepatocellular carcinoma; $\mathrm{HCV}$, hepatitis $\mathrm{C}$ virus; $\mathrm{MR}$, magnetic resonance.

Observations without definite hyperenhancement may be ultimately categorized as LR-3 or LR-4, but not as LR-5. ${ }^{17}$

\section{Diameter}

Diameter is defined as the largest diameter (outer edge to outer edge) measured in the sequence or phase in which the margins are most sharply demarcated and without apparent anatomic distortion. ${ }^{17}$ It is preferable to avoid measuring an observation in the arterial phase, as measurement in this phase is less reliable if the enhancement is incomplete due to an early arterial phase or if there is perilesional enhancement. Diameter, as a major imaging feature, is stratified into four size categories:

Diameter $<20 \mathrm{~mm}$ is used to further categorize masses with arterial-phase hypo- or isoenhancement. Masses that meet both of these criteria can be categorized as LR-3 or LR-4, depending upon other imaging features. They cannot, however, be categorized at LR-5. ${ }^{17}$

Diameter $<10 \mathrm{~mm}$ is used to further characterize masses with arterial-phase hyperenhancement. Masses that meet both of these criteria may be categorized as LR-3 or LR-4, depending upon the presence of other major or ancillary imaging features. ${ }^{16,17}$ These observations cannot be categorized as definite HCC (LR-5) because of the lower specificity of CT or MRI for nodules $<1 \mathrm{~cm} .{ }^{17}$

Diameter 10-19 $\mathrm{mm}$ is used to further categorize masses with arterial-phase hyperenhancement. Masses that meet both of these criteria may be categorized as LR-4 or LR-5, depending upon the presence of other major or ancillary imaging features. ${ }^{17}$ Two or more additional major features are required for these observations to reach LR-5. To be congruent with the American Association for the Study of Liver Diseases (AASLD) and United Network for Organ Sharing (UNOS)-Organ Procurement and Transplantation Network (OPTN) practice guidelines, ${ }^{22,23}$ observations that present both washout appearance and visibility at antecedent surveillance ultrasound (LR-5us) and observations that present $\geq 50 \%$ diameter increase in $\leq 6$ months (LR-5g) can also be assigned an LR-5 category.

Diameter $\geq 20 \mathrm{~mm}$ is used to further characterize masses with any type of arterial-phase enhancement. These masses may be categorized as LR-3, LR-4, or LR-5, depending upon other major or ancillary imaging features. ${ }^{17}$

\section{Washout appearance}

Washout appearance, as it is called in the two most recent versions of LI-RADS (v2013 and v2014), was previously known as "portal venous or later-phase hypoenhancement" in the 2011 version. This terminology is congruent with OPTN terminology. ${ }^{17}$ The term "washout appearance" is used because not all of these observations have true washout, as defined by reducing signal on enhancement curves; increased enhancement of background liver can contribute to washout appearance. Washout appearance is a strong predictor of HCC in arterially hyperenhancing hepatic observations (Figure 2). ${ }^{24}$ This phenomenon results from decreased portal venous supply to, and early venous drainage from, the HCC accompanying neoangiogenesis, in addition to increased late enhancement of fibrosis in the surrounding liver parenchyma during portal venous or delayed phases following extracellular contrast agent administration. ${ }^{25,26}$ Special care should be made to ensure that the degree of enhancement during these phases is unequivocally lower than during earlier phases, as well as making certain that the same observations are compared in different phases. ${ }^{27}$ Subtraction imaging may be helpful to assess washout. Rimola et $\mathrm{al}^{28}$ evaluated washout features with dynamic MRI in 5-20 mm observations detected by screening ultrasound of patients with cirrhosis. Though the sensitivity of washout was only $58.3 \%$, diagnosis of HCC in masses demonstrating arterial hyperenhancement with washout yielded specificity of $96.4 \%$ and a positive predictive value of $96.8 \% .^{28}$ How well these results generalize to observations that were not first detected by screening ultrasound is not well understood. 
Washout can be present as only part of an observation, which presents a potential pitfall. Washout in the periphery of an observation is considered "peripheral washout" and not "washout appearance." Peripheral washout suggests intrahepatic cholangiocarcinoma rather than HCC, making an observation fall into the LR-M category. ${ }^{17}$

Fibrosis may also create the false perception of washout. Fibrosis generally demonstrates increased enhancement on late post-contrast images, potentially creating an appearance of hypoenhancement of an encompassed regenerative nodule or mimicking a delayed enhancing capsule..$^{16,29}$

\section{Capsule appearance}

Capsule appearance is a major feature of HCC defined as a peripheral rim of smooth hyperenhancement in the portal venous or delayed phase that unequivocally is thicker and more conspicuous than the rims surrounding background nodules (Figure 2) ${ }^{28}$ Since some of these masses may not have a true capsule, the terms capsule appearance or "capsule" (with quotes) are preferred over capsule alone. Whether a mass has a true capsule or pseudocapsule can only be distinguished pathologically, and there are no data to support the diagnostic significance of this distinction. The degree of enhancement typically increases between the portal venous and delayed venous phases. Rimola et $\mathrm{a}^{28}$ found that capsule appearance provided a sensitivity, specificity, negative predictive value, and positive predictive value of $41.7 \%, 96.4 \%, 95.6 \%$, and $47.4 \%$, respectively.

\section{Threshold growth}

Threshold growth is a major feature that reflects shorter tumor volume doubling time of HCC compared to that of nonmalignant lesions. ${ }^{16}$ Threshold growth is defined as a diameter increase of a mass by at least $5 \mathrm{~mm}$ and by a $>50 \%$ increase in $\leq 6$ months, or $>100 \%$ in $>6$ months. A new mass $\geq 10 \mathrm{~mm}$ in size, regardless of time interval, is also considered as threshold growth. ${ }^{16}$ Of note, masses that demonstrate threshold growth but do not demonstrate arterial-phase hyperenhancement may not be categorized as LR-5. ${ }^{17}$ It is important to measure consistently in the same plane and, if possible, in the same sequence or phase. Again, the arterial phase is not ideal for measurement, as these measurements may be affected by the early timing of the image acquisition or perilesional enhancement. ${ }^{17}$

\section{Tumor in vein}

Vascular invasion of HCC occurs by direct invasion of the tumor into an adjacent portal, or less often, hepatic vein. ${ }^{30,31}$ Tumor in a vein, defined as definitive soft tissue enhancement within a vein, is now the preferred term over tumor thrombus. ${ }^{16}$
This feature - which is associated with a poor prognosis, modifies the staging, and may contraindicate eligibility for local ablative therapies and liver transplantation - is demonstrated by unequivocal luminal enhancement in the arterial phase with washout of the soft tissue component of the tumor. ${ }^{32}$ Tumors demonstrating this feature are classified as LR-5V, a definitive diagnosis of HCC (although indeed some cholangiocarcinomas show tumor extension into veins). ${ }^{17}$

Mimics of venous involvement include early enhancement of the portal veins related to late arterial-phase timing, the presence of arterioportal shunting, or hepatofugal flow. ${ }^{14}$ Similar findings in the hepatic veins can occur with arteriovenous shunting or reflux of contrast into the hepatic veins from the inferior vena cava. ${ }^{16}$ Thus, observation of washout after enhancement in the involved vein, greater than other vessels, is critical. In general, tumor in the vein will demonstrate a more heterogeneous appearance than shunted/ retrograde contrast flow (Figure 3). Another mimic of tumor thrombus is bland (chronic) thrombus, which also occurs in cirrhosis secondary to portal hypertension and venous stasis. ${ }^{31}$ In comparison to tumor in the vein, bland thrombus causes less expansion of the vessels, does not demonstrate contrast enhancement, and may exhibit low T2 signal related to hemosiderin when it is long standing. ${ }^{16,33}$ Cavernous transformation around a bland thrombus can serve as an additional complicating factor. ${ }^{16}$ Doppler ultrasound or tissue sampling may be helpful in determining the nature of a thrombus when tumor in vein versus bland thrombus are questioned.

\section{Ancillary imaging features}

Ancillary features, which can be used to upgrade or downgrade an observation by one or more categories, are left to the discretion of the radiologist. ${ }^{16}$ These features modify the likelihood of HCC but are currently not supported by sufficient evidence to categorize a finding independently. ${ }^{17}$ Ancillary features that favor HCC may be used to upgrade observations from one LIRADS category to the next, but such upgrades may go only as high as LR-4 and not to LR-5. Similarly, imaging features that favor benignity may be used to downgrade observations that otherwise meet major criteria for a higher category. ${ }^{16}$ Absence of ancillary features does not justify designation of a higher or lower category. ${ }^{17}$ Examples of ancillary features favoring HCC or benignity are listed in Table 3.

If, after application of ancillary features, a radiologist is still unsure about the final category for an observation, tiebreaking rules should be applied. Generally, tiebreaking rules move observations away from LR-1 and LR-5 toward LR-3 to maintain the highest degree of specificity for the ultimate diagnosis of HCC. ${ }^{16,17}$ 

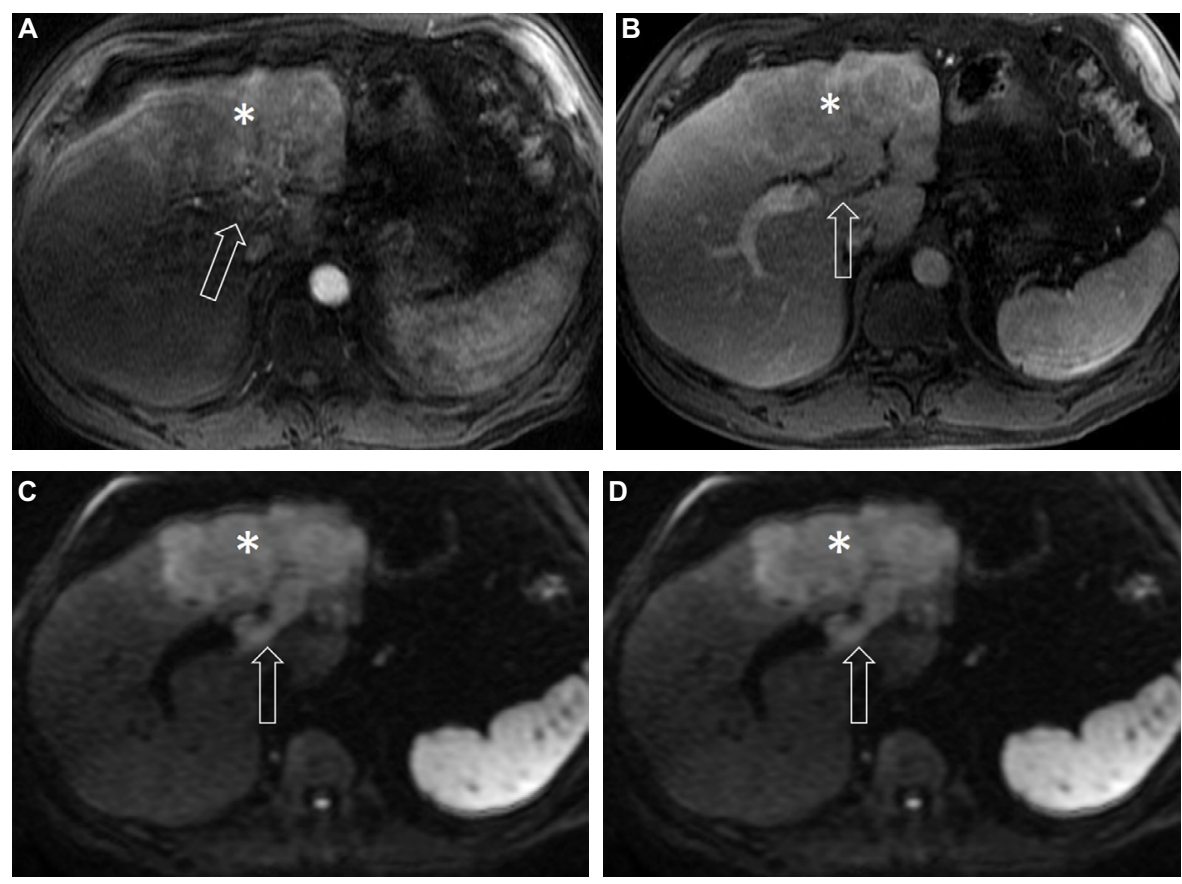

Figure $3 \mathrm{HCC}$ in a 67-year-old man with alcoholic cirrhosis.

Notes: Axial post-contrast TI-weighted MR images in arterial (A), delayed phase (B), axial T2-weighted (C), and diffusion weighted (D) MR images demonstrate an infiltrative mass (asterisk) with ill-defined margins, exhibiting heterogeneous enhancement in the arterial phase, washout in the delayed phase with moderately increased signal intensity on T2-weighted and diffusion restriction on diffusion-weighted images. There is soft tissue noted within the left portal vein (arrow) exhibiting all signal characteristics and contrast enhancement similar to the tumor, representing LR-5V. See Table 2 for LI-RADS classifications.

Abbreviations: HCC, hepatocellular carcinoma; MR, magnetic resonance.

\section{v20I4 categories}

LI-RADS categories, with corresponding descriptions and management guidance, are summarized in Table 2.

\section{Category LR-I}

Observations in this category are definitely benign, with $100 \%$ certainty. ${ }^{17}$ Unnecessary follow-up imaging may be avoided, and multidisciplinary discussion is not warranted. ${ }^{17}$ Lesions in LR-1 and LR-2 categories are summarized in Table 4.

\section{Category LR-2}

Findings in this category have a high probability, though not $100 \%$, of being benign. Most of these are benign processes

Table 3 Ancillary imaging features in favoring diagnosis of HCC and favoring benignity

\begin{tabular}{ll}
\hline Favor HCC & Favor benignity \\
\hline $\begin{array}{l}\text { Mild to moderate T2 hyperintensity } \\
\text { Subthreshold growth }\end{array}$ & $\begin{array}{l}\text { Uniform marked T2 hyperintensity } \\
\text { Uniform marked T2 hypointensity } \\
\text { Mosaic architecture }\end{array}$ \\
$\begin{array}{l}\text { Normal, undistorted vessels } \\
\text { crossing through region of interest }\end{array}$ \\
$\begin{array}{ll}\text { Fat deposition disproportionate to } \\
\text { that in the surrounding fat }\end{array}$ & $\begin{array}{l}\text { Blood pool enhancement } \\
\text { characteristics } \\
\text { Restricted diffusion }\end{array}$ \\
$\begin{array}{l}\text { Decrease in diameter of I0+ mm } \\
\text { without treatment }\end{array}$ \\
\hline
\end{tabular}

Abbreviation: $\mathrm{HCC}$, hepatocellular carcinoma. with an atypical appearance, such as slow-filling hemangiomas. ${ }^{17}$ Notably, focal perfusion alterations related to nonmalignant arterioportal shunts or portal venous branch obstruction are LR-2 observations, because small HCCs may occasionally have similar appearance. ${ }^{11,34}$ Imaging characteristics include peripheral, wedge-shaped, and exclusively arterial-enhancing observations that are isointense or near isointense on T1- and T2-weighted images. ${ }^{35}$ If not all of these criteria are met, the observation should be considered LR-3 or LR-4. ${ }^{36}$ Particular caution is indicated here because perfusion alteration can occur secondary to focal hepatic observations, such as HCC. ${ }^{34}$

Some other examples of LR-2 observations are vascular anomalies, perfusion alterations, hepatic fat deposition or sparing and focal scars, among others. Hepatic adenoma and focal nodular hyperplasia (FNH) are generally not considered

Table 4 Examples of definitely benign (category LR-I) and probably benign (category LR-2) lesions

\begin{tabular}{ll}
\hline $\begin{array}{l}\text { Definitely benign; category } \\
\text { LR-I }\end{array}$ & $\begin{array}{l}\text { Probably benign; category } \\
\text { LR-2 }\end{array}$ \\
\hline Cyst, hemangioma, focal fat & Atypical presentation of cyst, \\
deposition or sparing, confluent & hemangioma, focal fat deposition \\
fibrosis, perfusion anomalies, & or sparing, confluent fibrosis, \\
focal scars, or nonhyperenhancing & perfusion anomalies, focal scars, or \\
arterial nodules & nonhyperenhancing arterial nodules \\
\hline
\end{tabular}


LR-1 or LR-2, as these entities are rare in cirrhosis and, even when present, cannot be confidently distinguished from HCC in high-risk individuals. ${ }^{17,37,38}$ v2014 also does not address regenerative nodules occurring in the absence of cirrhosis, such as those associated with Budd-Chiari syndrome. ${ }^{17}$

\section{Category LR-3}

Observations in this category have an intermediate probability of being HCC. LR-3 includes all observations that lack both unequivocal major features of LR-4 and LR-5 and unequivocal benign features of LR-1 and LR-2. ${ }^{16}$ They do not definitively fit into any other LI-RADS category. ${ }^{17}$ LR-4 and LR-5 observations that are stable in size and appearance over 2 years are considered LR-3. The American Association for the Study of Liver Disease recommends that findings categorized as LR-3 and $<10 \mathrm{~mm}$ be reimaged in 3-6 months. Three-month follow-up with contrast-enhanced CT or MRI is preferred over biopsy for further workup of observations $>10 \mathrm{~mm} .^{11}$

\section{Category LR-4}

Observations in this category have a high probability of HCC and demonstrate some, but not all, major imaging features. ${ }^{16}$ LR-4 observations are not definitely or probably benign, do not qualify as non-HCC malignancy, and do not include tumor in the vein. ${ }^{17}$ LR-4 was previously subdivided into category A ( $<20 \mathrm{~mm})$ and category $\mathrm{B}(>20 \mathrm{~mm})$, with one additional major feature required for LR-4 assignment to category A. ${ }^{16}$ This subdivision has been discontinued in the v2014 classification since reporting of observation diameter is required. Probable tumor in vein is also considered LR-4, as any uncertainty between LR-4 and LR-5 category assignment should be given LR-4 status. ${ }^{17}$ As they represent probable HCC, LR-4 observations require close follow-up and may need additional imaging or biopsy. ${ }^{17}$

\section{Category LR-5}

Observations in this category are definitely HCC. ${ }^{16,17}$ This designation should be used only when imaging criteria are unequivocal and sufficient to render a diagnosis of HCC with absolute certainty without a biopsy. ${ }^{16}$ LR-5 was previously subdivided on the basis of size into categories A (between 10 and $20 \mathrm{~mm})$ and $\mathrm{B}(>20 \mathrm{~mm})$. This subcategorization has now been removed in $\mathrm{v} 2014$.

A 10-19 mm observation requires arterial-phase hyperenhancement and at least two of the following major criteria: washout, capsule, or threshold growth, to be categorized as LR-5. Observations that present both washout appearance and visibility at antecedent surveillance ultrasound (LR-5us) and observations that present $\geq 50 \%$ diameter increase in $\leq 6$ months (LR-5g) are also categorized as LR-5.
Observations $\geq 20 \mathrm{~mm}$ with arterial-phase hyperenhancement require at least one of the following major criteria: washout, capsule, or threshold growth, to be categorized as LR-5.

\section{Category LR-5V}

Observations with features of definite venous invasion are LR-5V, regardless of the presence of other major features. ${ }^{17}$

\section{LR treated}

The LR-treated category is new with the implementation of v2014. Any observation that has undergone locoregional treatment is placed into this category, regardless of whether or not treatment was successful. ${ }^{17}$ Residual or recurrent HCC may be present. ${ }^{17}$ Criteria for assessing treatment response are still being developed and will appear in the 2017 update. ${ }^{17}$ Notably, observations undergoing systemic treatment are not classified using LR treated. ${ }^{17}$

\section{LI-RADS and liver transplantation}

In patients with cirrhosis and $\mathrm{HCC}$, hepatic transplantation is the treatment with the highest 5-year reported survival rate of $84 \%$ as compared to $46 \%$ and $34 \%$ for resection and ablation, respectively. ${ }^{11}$ The United Network for Organ Sharing governs the OPTN, which maintains a national transplant waiting list. The policy of these organizations is to assess mortality risk using the Model for End-Stage Liver Disease (MELD) score to assess transplant priority. ${ }^{22,35}$ Diagnosis of HCC does not require tissue diagnosis if OPTN class 5 imaging criteria for HCC are met on multiphasic post-contrast MRI or CT that meet minimum technical requirements. ${ }^{39}$

\section{OPTN classification system}

OPTN uses a distinct terminology for description of HCCs (Table 5). OPTN class 5 indicates that a nodule meets radiologic criteria for HCC. Class $5 \mathrm{~A}$ refers to a single nodule $\geq 1 \mathrm{~cm}$ and $<2 \mathrm{~cm}$ in diameter with arterial-phase hyperenhancement and has at least one of two venous features of HCC (washout and capsule appearance). Class 5A-g (for growth) applies to a single nodule $\geq 1 \mathrm{~cm}$ and $<2 \mathrm{~cm}$ in diameter with arterial-phase hyperenhancement and has growth by $\geq 50 \%$ on MRI or CT obtained $\leq 6$ months apart. ${ }^{23,39}$ Class 5B applies to a single nodule $\geq 2 \mathrm{~cm}$ and $\leq 5 \mathrm{~cm}$ with arterial-phase hyperenhancement and one of the following: washout on portal venous or delayed phase, capsule appearance, growth by $\geq 50 \%$ documented on serial MRI or CT obtained $\leq 6$ months apart. Class 5T applies to class 5 lesions that were previously treated by locoregional ablation. Class $5 \mathrm{X}$ refers to lesions that meet radiologic criteria for $\mathrm{HCC}$ but are $\geq 5 \mathrm{~cm} .^{22,23,39}$ 
Table 5 OPTN classification system for nodules seen on images of cirrhotic livers

\begin{tabular}{ll}
\hline Class and description & Comment \\
\hline OPTN class 0: incomplete or technically inadequate study & $\begin{array}{l}\text { Repeat study required for adequate assessment; automatic MELD priority points } \\
\text { cannot be assigned on basis of an imaging study categorized as OPTN class } 0\end{array}$ \\
OPTN class 5: meets radiologic criteria for HCC & $\begin{array}{l}\text { May qualify for automatic exception depending on stage } \\
\text { Increased arterial enhancement during the late hepatic arterial phase and washout } \\
\text { during the later phases of contrast enhancement and peripheral rim enhancement } \\
\text { (capsule or pseudocapsule) }\end{array}$ \\
OPTN class 5A: lesion $\geq 1 \mathrm{~cm},<2 \mathrm{~cm}$ measured in late & $\begin{array}{l}\text { Increased contrast enhancement in the late hepatic arterial phase and growth by } \geq 50 \% \\
\text { arterial or portal venous phase images }\end{array}$ \\
OPTN class 5A-g: same size as OPTN class 5A HCC & $\begin{array}{l}\text { Increased contrast enhancement in the late hepatic arterial phase and either } \\
\text { washout during later contrast phases or peripheral rim enhancement (capsule or } \\
\text { pseudocapsule), 50\% growth or more documented on serial CT or MR images } \\
\text { obtained } 6 \text { months apart (OPTN class 5B-g) }\end{array}$ \\
Any residual lesion or perfusion defect at site of prior UNOS class 5 lesion \\
Increased contrast enhancement in the late hepatic arterial phase and either washout \\
OPTN class 5T: prior regional treatment for HCC & during later contrast phases or peripheral rim enhancement (capsule or pseudocapsule)
\end{tabular}

Notes: Data from OPTN/UNOS Liver and Intestinal Organ Transplantation Committee. Report to the Board of Directors; 2016. Available from: http://optn.transplant.hrsa. gov. ${ }^{37}$ Reproduced, with permission, from Wald C, Russo MW, Heimbach JK, Hussain HK, Pomfret EA, Bruix J. New OPTN/UNOS policy for liver transplantation allocation: standardization of liver imaging, diagnosis, classification, and reporting of hepatocellular carcinoma. Radiology. 2013;266(2):376-382. ${ }^{23}$

Abbreviations: HCC, hepatocellular carcinoma; CT, computed tomography; MR, magnetic resonance; UNOS, United Network for Organ Sharing; OPTN, Organ Procurement and Transplantation Network; MELD, Model for End-Stage Liver Disease.

\section{Eligibility for liver transplantation}

To qualify for MELD exception points, liver transplant candidates with HCC must have radiologic stage 2 disease at the time of initial prioritization, which implies either, 1) one HCC measuring $\geq 2 \mathrm{~cm}$ and $\leq 5 \mathrm{~cm}$ in diameter, or 2) up to three HCCs, each $\geq 1 \mathrm{~cm}$ and $\leq 3 \mathrm{~cm}$ in diameter.

Patients beyond Milan criteria or radiologic stage 2 disease must undergo downstaging to $\mathrm{T} 2$ before being considered for liver transplantation. These patients must go through the regional review board for determination of eligibility and do not receive automatic MELD exception points.

Regardless of size and number of HCCs, eligibility always requires absence of extrahepatic involvement and macrovascular invasion. ${ }^{11,23}$

\section{LI-RADS versus OPTN}

LI-RADS is used for characterization of all liver nodules from benign nodules to HCC, while the updated OPTN criteria (OPTN class 5) are used only to characterize HCC and determine eligibility for MELD exception points for the purpose of prioritization of transplant candidates with HCC. ${ }^{22,23,39}$ v2014 was created for congruency with OPTN-5 criteria. Therefore, in the most updated version of LI-RADS, LR-5 and OPTN-5 are very similar (Table 6). ${ }^{16}$ However, LR-1 to LR-4 are not part of the OPTN criteria. ${ }^{17}$

\section{LI-RADS version 2017}

It is anticipated that LI-RADS will be updated in 2017 and 2020 through the ACR. Each future iteration will incorporate

Table 6 Comparison of OPTN class 5 and LI-RADS category 5

\begin{tabular}{|c|c|c|}
\hline Size & OPTN classification & LI-RADS category \\
\hline $\mathrm{I}-2 \mathrm{~cm} \mathrm{HCC}$ & $\begin{array}{l}\text { OPTN class } 5 \mathrm{~A}: \geq 1 \mathrm{~cm},<2 \mathrm{~cm} \text { measured in late arterial or portal venous } \\
\text { phase images. Increased arterial enhancement during the late hepatic arterial } \\
\text { phase and washout during the later phases of contrast enhancement and } \\
\text { peripheral rim enhancement (capsule or pseudocapsule). } \\
\text { OPTN class } 5 \mathrm{~A}-\mathrm{g} \text { : increased contrast enhancement in the late hepatic } \\
\text { arterial phase and growth by } \geq 50 \% \text { documented on serial CT or MR images } \\
\text { obtained } \leq 6 \text { months apart. }\end{array}$ & $\begin{array}{l}\text { LR-5: } 10-19 \mathrm{~mm} \text { mass with arterial-phase } \\
\text { hyperenhancement and } \geq 2 \text { of the following: washout } \\
\text { appearance, capsule appearance, or threshold growth. }\end{array}$ \\
\hline$\geq 2 \mathrm{~cm} \mathrm{HCC}$ & $\begin{array}{l}\text { OPTN class B: increased contrast enhancement in late hepatic arterial } \\
\text { phase and either washout during later contrast phases or peripheral rim } \\
\text { enhancement (capsule or pseudocapsule). }\end{array}$ & $\begin{array}{l}\text { LR-5: } \geq 20 \mathrm{~mm} \text { mass with arterial-phase } \\
\text { hyperenhancement and } \geq 1 \text { of the following: washout } \\
\text { appearance, capsule appearance, or threshold growth. }\end{array}$ \\
\hline $\begin{array}{l}\text { HCC with } \\
\text { tumor in vein }\end{array}$ & $\begin{array}{l}\text { Imaging not provided as patients with tumor in vein are not eligible for liver } \\
\text { transplant. }\end{array}$ & $\begin{array}{l}\text { LR-5V: HCC with tumor in vein; definite enhancing } \\
\text { tissue in vein. }\end{array}$ \\
\hline
\end{tabular}

Notes: See Table 2 for LI-RADS classification and Table 5 for OPTN classification system for nodules seen on images of cirrhotic livers. Data from OPTN/UNOS Liver and Intestinal Organ Transplantation Committee. Report to the Board of Directors; 2016. Available from: http://optn.transplant.hrsa.gov37 and Wald et al.23

Abbreviations: LI-RADS, Liver Imaging Reporting and Data System; HCC, hepatocellular carcinoma; CT, computed tomography; MR, magnetic resonance; OPTN, Organ Procurement and Transplantation Network. 
new published evidence as well as patient outcomes in order to improve upon the existing system. In 2017, some of the important expansions include development of LI-RADS characterization of findings on ultrasound screening studies. Contrast-enhanced ultrasound will also be incorporated into LI-RADS for characterizing visible lesions identified with this type of cross-sectional imaging modality. There is a working group within LI-RADS that is helping to incorporate hepatobiliary phase agents into interpretation of liver observations. An expansion of evaluation of LR-treated lesions will also be provided, including how to interpret findings and report tumors after locoregional therapy. New refinements of ancillary categories will be provided, in a user-friendly format, based on incorporation of published data as well as outcomes of consensus meetings, which continue to include radiologists, hepatologists, hepatobiliary surgeons, and interventional radiologists. Improved standardization of image quality will be included as well as guidelines of how to categorize observations in the liver when imaging quality is suboptimal for technical or patientrelated reasons. The LI-RADS community continues to liaise with OPTN and AASLD in order to ensure that radiologists are able to provide value-added reports, which will help surgeons and clinicians efficiently and effectively serve their patients.

\section{Conclusion}

LI-RADS system, first created in 2011, is a consensus ACRsupported initiative analogous to BI-RADS in breast imaging. LI-RADS has helped to increase clarity, consistency, and quality of diagnostic reports and thereby improved patient care. LI-RADS is a dynamic process, which is updated about every 3 years as imaging techniques improve, current content ambiguities are resolved, and user feedback is collected. Various components of v2014 were described in this article. LI-RADS will be updated in 2017 through the ACR and each future iteration will incorporate new evidence related to radiological knowledge and patient outcomes in order to improve upon the existing system.

\section{Disclosure}

The authors report no conflicts of interest in this work.

\section{References}

1. Parkin DM, Bray F, Ferlay J, Pisani P. Estimating the world cancer burden: Globocan 2000. Int J Cancer. 2001;94(2):153-156.

2. Dhanasekaran R, Limaye A, Cabrera R. Hepatocellular carcinoma: current trends in worldwide epidemiology, risk factors, diagnosis, and therapeutics. Hepat Med. 2012;4:19-37.

3. El-Serag HB. Hepatocellular carcinoma: recent trends in the United States. Gastroenterology. 2004;127(5 Suppl 1):S27-S34.

4. El-Serag HB, Mason AC. Rising incidence of hepatocellular carcinoma in the United States. N Engl J Med. 1999;340(10):745-750.
5. Thompson Coon J, Rogers G, Hewson P, et al. Surveillance of cirrhosis for hepatocellular carcinoma: systematic review and economic analysis. Health Technol Assess. 2007;11(34):1-206.

6. Llovet JM, Burroughs A, Bruix J. Hepatocellular carcinoma. Lancet. 2003;362(9399):1907-1917.

7. El-Serag HB, Rudolph KL. Hepatocellular carcinoma: epidemiology and molecular carcinogenesis. Gastroenterology. 2007;132(7): 2557-2576.

8. Marrero JA, Fontana RJ, Su GL, Conjeevaram HS, Emick DM, Lok AS. NAFLD may be a common underlying liver disease in patients with hepatocellular carcinoma in the United States. Hepatology. 2002;36(6): 1349-1354.

9. Liaw YF, Tai DI, Chu CM, et al. Early detection of hepatocellular carcinoma in patients with chronic type B hepatitis: a prospective study. Gastroenterology. 1986;90(2):263-267.

10. Bosch FX, Ribes J, Diaz M, Cleries R. Primary liver cancer: worldwide incidence and trends. Gastroenterology. 2004;127(5 suppl1):S5-S16.

11. Bruix J, Sherman M; Practice Guidelines Committee, American Association for the Study of Liver Diseases. Management of hepatocellular carcinoma. Hepatology. 2005;42(5):1208-1236.

12. Zhang BH, Yang BH, Tang ZY. Randomized controlled trial of screening for hepatocellular carcinoma. J Cancer Res Clin Oncol. 2004; 130(7):417-422.

13. Chou R, Cuevas $\mathrm{C}, \mathrm{Fu} \mathrm{R}$, et al. Imaging techniques for the diagnosis of hepatocellular carcinoma: a systematic review and meta-analysis. Ann Intern Med. 2015;162(10):697-711.

14. Altekruse SF, McGlynn KA, Dickie LA, Kleiner DE. Hepatocellular carcinoma confirmation, treatment, and survival in surveillance, epidemiology, and end results registries, 1992-2008. Hepatology. 2012;55(2): 476-482.

15. Jeong YY, Nim NY, Kang HK. Hepatocellular carcinoma in the cirrhotic liver with helical CT and MRI: imaging spectrum and pitfalls in cirrhosis-related nodules. AJR Am J Roentgenol. 2005;185:1024-1032.

16. American College of Radiology [webpage on the Internet]. Liver Imaging Reporting and Data System version 2013. Available from: http:// www.acr.org/Quality-Safety/Resources/LIRADS. Accessed November 7, 2016.

17. American College of Radiology. Liver Imaging and Reporting System version 2014. Available from: https://nrdr.acr.org/lirads/. Accessed November 23, 2016.

18. Willatt JM, Hussain HK, Adusumilli S, Marrero JA. MR Imaging of hepatocellular carcinoma in the cirrhotic liver: challenges and controversies. Radiology. 2008;247(2):311-330.

19. Sharma P, Kalb B, Kitajima HD, et al. Optimization of single injection liver arterial phase gadolinium enhanced MRI using bolus track realtime imaging. J Magn Reson Imaging. 2011;33(1):110-111.

20. Ayuso C, Rimola J, García-Criado A. Imaging of HCC. Abdom Imaging. 2012;37(2):215-230.

21. Efremidis SC, Hytiroglou P. The multistep process of hepatocarcinogenesis in cirrhosis with imaging correlation. Eur Radiol. 2002;12(4): 753-764.

22. OPTN/UNOS Liver and Intestinal Organ Transplantation Committee [homepage on the Internet]. Report to the Board of Directors; 2016. Available from: http://optn.transplant.hrsa.gov. Accessed November 2016.

23. Wald C, Russo MW, Heimbach JK, Hussain HK, Pomfret EA, Bruix J. New OPTN/UNOS policy for liver transplantation allocation: standardization of liver imaging, diagnosis, classification, and reporting of hepatocellular carcinoma. Radiology. 2013;266(2):376-382.

24. Marrero JA, Hussain HK, Nghiem HV, Umar R, Fontana RJ, Lok AS. Improving the prediction of hepatocellular carcinoma in cirrhotic patients with an arterially-enhancing liver mass. Liver Transpl. 2005;11(3): 281-289.

25. Kadoya M, Matsui O, Takashima T, Nonomura A. Hepatocellular carcinoma: correlation of MR imaging and histopathologic findings. Radiology. 1992;183(3):819-825.

26. Sherman M. The radiological diagnosis of hepatocellular carcinoma. Am J Gastroenterol. 2010;105(3):610-612. 
27. PuryskoAS, RemerEM, Coppa CP, Leão Filho HM, ThupiliCR, Veniero JC LI-Rads: a case based review of the new categorization of liver findings in patients with end stage liver disease. Radiographics. 2012;32:1977-1995.

28. Rimola J, Alejandro F, Tremosini S, et al. Non-invasive diagnosis of hepatocellular carcinoma $\leq 2 \mathrm{~cm}$ in cirrhosis. Diagnostic accuracy assessing fat, capsule and signal intensity at dynamic MRI. J Hepatol. 2012; 56(6):1317-1323.

29. Fowler KJ, Brown JJ, Narra VR. Magnetic resonance imaging of focal liver lesions: approach to imaging diagnosis. Hepatology. 2011;54(6): 2227-2237.

30. Ueda K, Matsui O, Kawamori Y, et al. Hypervascular hepatocellular carcinoma: evaluation of hemodynamics with dynamic CT during hepatic arteriography. Radiology. 1998;206(1):161-166.

31. Dodd GD 3rd, Memel DS, Baron RL, Eichner L, Santiguida LA. Portal vein thrombosis in patients with cirrhosis: does sonographic detection of intrathrombus flow allow differentiation of benign and malignant thrombus? AJR Am J Roentgenol. 1995;165(3):573-577.

32. Sakata J, Shirai Y, Wakai T, Kaneko K, Nagahashi M, Hatakeyama K Preoperative predictors of vascular invasion in hepatocellular carcinoma. Eur J Surg Oncol. 2008;34(8):900-905.
33. Willatt JM, Hussain HK, Saroja A, Marrero J. MR Imaging of hepatocellular carcinoma in the cirrhotic liver: challenges and controversies. Radiology. 2008;247(2):311-330.

34. Colagrande S, Centi N, Galdiero R, Ragozzino A. Transient hepatic intensity differences. 1. Those associated with focal lesions. AJR Am J Roentgenol. 2007;188(1):154-159.

35. Brancatelli G, Baron RL, Peterson MS, Marsh W. Helical CT screening for hepatocellular carcinoma in patients with cirrhosis: frequency and causes of false-positive interpretation. AJR Am J Roentgenol. 2003; 180(4):1007-1014.

36. Jeong YY, Mitchell DG, Kamishima T. Small $(<20 \mathrm{~mm})$ enhancing hepatic nodules seen on arterial phase MR imaging of the cirrhotic liver: clinical implications. AJR Am J Roentgenol. 2002;178(6):1327-1334.

37. Hussein SM, Reinhold C, Mitchell DG. Cirrhosis and lesion characterization at MR imaging. Radiographics. 2009;29:1637-1652.

38. Lee JM, Yoon JH, Joo I, et al. Recent advances in CT and MR imaging for hepatocellular carcinoma. Liver Cancer. 2012;1(1):22-40.

39. Mazzaferro V, Regalia E, Doci R, et al. Liver transplantation for the treatment of small hepatocellular carcinomas in patients with cirrhosis N Engl J Med. 1996;334(11):693-700.
Journal of Hepatocellular Carcinoma

\section{Publish your work in this journal}

The Journal of Hepatocellular Carcinoma is an international, peerreviewed, open access journal that offers a platform for the dissemination and study of clinical, translational and basic research findings in this rapidly developing field. Development in areas including, but not limited to, epidemiology, vaccination, hepatitis therapy, pathology and

\section{Dovepress}

molecular tumor classification and prognostication are all considered for publication. The manuscript management system is completely online and includes a very quick and fair peer-review system, which is all easy to use. Visit http://www.dovepress.com/testimonials.php to read real quotes from published authors. 